\title{
CORRECTION
}

\section{Correction to: Electromagnetic interference in implantable cardioverter defibrillators: present but rare}

\author{
Gesa von Olshausen ${ }^{1} \cdot$ Ina-Christine Rondak ${ }^{2} \cdot$ Carsten Lennerz $^{3} \cdot$ Verena Semmler $^{3} \cdot$ Christian Grebmer $^{3}$. \\ Tilko Reents ${ }^{3}$. Sonia Ammar-Busch ${ }^{3} \cdot$ Alessandra Buiatti $^{3} \cdot$ Felix Bourier $^{3} \cdot$ Isabel Deisenhofer $^{3} \cdot$ Christof Kolb $^{3}$
}

Published online: 17 January 2020

(c) Springer-Verlag GmbH Germany, part of Springer Nature 2020

\section{Correction to: Clin Res Cardiol (2016) 105:657-665 https://doi.org/10.1007/s00392-016-0965-1}

The original version of this article unfortunately contained a mistake.

Section "Conclusion", first sentence should read: In a large cohort of ICD recipients clinically significant or potentially significant episodes of EMI occurred with an overall incidence of $0.27 \%$ and a non-hospital incidence of $0.04 \%$ per patient-year.

The original article can be found online at https://doi.org/10.1007/ s00392-016-0965-1.

Gesa von Olshausen

gesa.olshausen@tum.de

1 Medizinische Klinik, Klinikum rechts der Isar, Technische Universität München, Munich, Germany

2 Institut für Medizinische Statistik und Epidemiologie, Technische Universität München, Munich, Germany

3 Klinik für Herz- und Kreislauferkrankungen, Deutsches Herzzentrum München, , Technische Universität München, Munich, Germany 